\title{
Clinical characteristics, virulence factors and molecular typing of methicillin-resistant Staphylococcus aureus infections in Shenzhen City, China
}

\author{
L. $\mathrm{HU}^{1,2} \dagger, \mathrm{Y} . \mathrm{LI}^{2} \dagger, \mathrm{Y} . \mathrm{LU}^{2}, \mathrm{~J} . \mathrm{D} . \mathrm{KLENA}^{3,4}, \mathrm{Y} . \mathrm{QIU}^{2}, \mathrm{Y} . \mathrm{LIN}^{2}, \mathrm{M} . \mathrm{JIANG}^{2}$, \\ X. $\mathrm{SHI}^{2}, \mathrm{~L} . \mathrm{CHEN}^{2}, \mathrm{X} . \mathrm{LIU}^{2}, \mathrm{H} . \mathrm{MA}^{2}, \mathrm{~J} . \mathrm{CHENG}^{2}, \mathrm{~S} . \mathrm{WU}^{3}$, \\ B. $\mathrm{KAN}^{5}$ AND Q. $\mathrm{HU}^{1,2 *}$ \\ ${ }^{1}$ College of Life Sciences, Shenzhen University, Shenzhen, Guangdong, China \\ ${ }^{2}$ Shenzhen Major Infectious Disease Control Key Laboratory, Shenzhen Center for Disease Control and \\ Prevention, Shenzhen, Guangdong Province, China \\ ${ }^{3}$ United States Centers for Disease Control and Prevention, Beijing, China \\ ${ }^{4}$ Division of Global Health Protection, Center for Global Health, United States Centers for Disease Control and \\ Prevention, Atlanta, GA, USA \\ ${ }^{5}$ Key Laboratory of Surveillance and Early-warning on Infectious Disease, Division of Infectious Disease, Chinese \\ Center for Disease Control and Prevention, Beijing, China
}

Received 11 March 2016; Final revision 23 June 2016; Accepted 24 June 2016; first published online 22 July 2016

\section{SUMMARY}

Methicillin-resistant Staphylococcus aureus (MRSA) has emerged as a serious hospital and community-acquired infection and some strains are associated with greater severity. We investigated the clinical variability and molecular characteristics of MRSA infections in Shenzhen, China through a study at nine sentinel hospitals from January to December 2014. MRSA infections were classified as community-associated (CA-MRSA), healthcare-associated (HA-MRSA), and healthcareassociated community-onset (HACO-MRSA). In total, 812 MRSA isolates were collected and 183 of these were selected for further study. Patients with HA-MRSA infections were generally of greater age compared to other groups. Distinct body site and clinical presentations were evident in infected patients, e.g. CA-MRSA (skin and soft tissue, 53\%), HA-MRSA (respiratory tract, 22\%; surgical site, $20 \%$; trauma wounds, $20 \%$ ) and HACO-MRSA (mastitis, $47 \%$ ). In contrast to HA-MRSA, other categories of strains were significantly more susceptible to gentamicin, sulfamethoxazole/ trimethoprim, and tetracycline. No resistance to vancomycin or linezolid was recorded. The predominant clonal lineage within each strain category was CC59-t437-SCCmec IV/V-agr I (CA, $51.4 \%$; HA, $28.9 \%$; HACO, $52.9 \%$ ) which exhibited characteristics of a traditional CA clone together with $a g r$ I which is more often associated with HA clones. In conclusion, for the three categories of MRSA infections, there were significant differences in clinical characteristics of patients, but the predominant clone in each category shared a similar genetic background which suggests that transmission of MRSA strains has occurred between the community and hospitals in Shenzhen.

Key words: CA-MRSA, clinical characteristics, HA-MRSA, HACO-MRSA, methicillin-resistant Staphylococcus aureus, molecular typing, virulence factors.

\footnotetext{
* Author for correspondence: Dr Q. Hu, Shenzhen Centre for Disease Control and Prevention, Shenzhen 518055, Guangdong Province, China.

(Email: huqinghua03@163.com)

$\dagger$ These authors contributed equally to this work.
}

\section{INTRODUCTION}

Methicillin-resistant Staphylococcus aureus (MRSA) is an important human and animal pathogen capable 
of causing serious outbreaks of infection in both hospital and community patients worldwide [1]. Healthcare-associated MRSA (HA-MRSA) infections are most often associated with surgery, dialysis, hospitalization, use of indwelling catheters or other percutaneous medical devices, and residence in a long-term care facility [2]. In the 1990s, reports appeared of community-acquired infections with MRSA (CA-MRSA) in healthy individuals such as army recruits, sports teams, and prisoners [3] and many of these infections were attributed to genetically novel strains [4]. More recently, a third category of MRSA has been recognized to describe strains that had the genetic characteristics of HA-MRSA but with community onset of infection (HACO-MRSA); the standard epidemiological definitions of infection categories was established by the US Centers for Disease Control and Prevention (US CDC) [5].

Several studies have shown that isolates causing CA- and HA-MRSA infections are distinct in antimicrobial resistance patterns, clinical and molecular characteristics [6], with HA-MRSA strains being typically more resistant to non- $\beta$-lactam antimicrobials [7]. Moreover, the staphylococcal cassette chromosome mec (SCCmec) elements conferring methicillin resistance in traditional HA-MRSA strains are most frequently of types I, II, or III whereas SCCmec elements IV and V, which are shorter and more easily transferred to other strains, are more often associated with CA-MRSA strains [8]. In addition, the accessory gene regulator (agr) type III is reported to be common in CA-MRSA strains, whereas HA-MRSA strains are more likely to be $a g r$ I or II [6]. However, it is becoming clear that the line between traditional HA-MRSA and CA-MRSA strains based on clinical characterization and susceptibility testing is becoming increasingly blurred [9], with some authors arguing in favour of infection category definitions according to the strain genotype isolated from the patient [10]. These reports imply that it is insufficient to base the definition of MRSA strains only on clinical characteristics.

Against this background we set out to determine the prevalence and clinical characteristics of patients presenting with MRSA infections in both the hospital and community in Shenzhen, a major city in Guangdong Province, located in the southeast of China. Correlates were sought between category of infection according to epidemiological criteria and molecular characteristics and genetic background of MRSA recovered within each infection category.

\section{METHODS}

\section{Bacterial isolation}

Nine sentinel hospitals were included in the project based on their geographical location, population diversity served, and laboratory capacity (Supplementary Fig. S1). These public hospitals serve $2500-6000$ outpatients per day and 1000-6500 inpatients per month. In each hospital, clinical specimens were cultured on blood agar and typical staphylococcal colonies were confirmed as $S$. aureus using the Vitek-2 Compact system (bioMérieux, USA). Isolates were screened for resistance to methicillin or oxacillin and MRSA were forwarded to the Shenzhen Center for Disease Control and Prevention (Shenzhen CDC) for further genetic characterization (Supplementary Fig. S2).

\section{Clinical data}

Clinical staff in the infection control department of the sentinel hospitals were responsible for collection of information on all MRSA-infected patients using a standard questionnaire to record demographic data, clinical information, and medical history.

\section{Case definition}

MRSA infections were categorized as HA, CA or HACO according to established criteria [5]. A patient was considered to be colonized with MRSA if the organism was isolated from sputum or throat swabs, but was apyrexial, did not have a raised white cell count or a positive chest X-ray.

\section{Antimicrobial susceptibility}

Minimal inhibitory concentrations of gentamicin, ciprofloxacin, sulfamethoxazole-trimethoprim, erythromycin, vancomycin, clindamycin, tetracycline and linezolid were determined in the Vitek-2 Compact system with AST-GP67 susceptibility cards (bioMérieux) according to the Clinical and Laboratory Standards Institute (CLSI) [11]. ATCC29213 (S. aureus) and ATCC29212 (Enterococcus faecalis) were used as reference controls.

\section{Virulence genes}

Isolates were screened for the $S$. aureus-specific thermonuclease gene $($ nuc), methicillin resistance gene mecA, Panton-Valentine leukocidin (PVL), and 16 
enterotoxin genes (sea, seb, sec, sed, see, seg, seh, sei, sej, sek, sel, sem, sen, seo, sep, seq) by polymerase chain reaction (PCR) using conserved primers as described previously and listed in Supplementary Table S1 $[12,13]$. DNA was extracted by suspending a single colony in $200 \mu \mathrm{l}$ of $2 \%(\mathrm{w} / \mathrm{v})$ Chelex 100 resin slurry (Bio-Rad Laboratories Ltd, Canada) and heating at $95^{\circ} \mathrm{C}$ for $10 \mathrm{~min}$. The presence of the $n u c$, mec $\mathrm{A}$ and $\mathrm{PVL}$ genes was determined via a triplex real-time PCR assay, and enterotoxin genes were tested by a single PCR.

\section{Molecular typing}

Isolates were characterized by SCCmec type, multilocus sequence type (MLST), staphylococcal protein A gene (spa) type, and accessory gene regulator (agr) using primers as in previous reports listed in Supplementary Table S1 [14-17]. Two multiplex PCR strategies were used to identify SCCmec types $\mathrm{I}-\mathrm{V}$; spa typing was performed in a single PCR and types were assigned using BioNumerics v. 6.0 software (Applied Maths, Belgium), and each of the four agr types was determined by a single PCR. Analysis of allelic profiles and sequence types (STs) were as described on the MLST website (http://saureus.mlst. net/), and eBURST v. 3.0 software (http://eburst. mlst.net/) was used to identify clonal complexes (CCs). Pulsed-field gel electrophoresis (PFGE) typing was based on $S m a I$ restriction profiles as described previously [18] and gel images were analysed using BioNumerics v. 6.0 software. Strain profiles were grouped using Dice coefficients and a $1.5 \%$ bandmatching criterion ( $80 \%$ relatedness) to define PFGE type. XbaI-digested Salmonella Braenderup H9812 DNA was used as a size standard.

\section{Data analysis}

Statistical analysis was performed using SPSS v. $16 \cdot 0$ software (SPSS Inc., USA). Demographic, clinical and genetic characteristics of different types of MRSA were compared using $\chi^{2}$ or Fisher's exact tests. $P$ values $<0 \cdot 05$ were considered statistically significant.

\section{Ethics}

The study was approved by the Institutional Review Board at Shenzhen CDC and the US CDC. All participants gave oral consent for use of their specimens and information for research purposes.

\section{RESULTS}

From January to December 2014, a total of 107406 clinical specimens were cultured for bacteria at the nine study hospitals, of which $3029(2 \cdot 8 \%)$ were identified as $S$. aureus and $812(0 \cdot 8 \%)$ as MRSA. Of these 812 strains, $596(73.4 \%)$ were excluded as colonizing strains and a further $33(4 \cdot 1 \%)$ could not be classified due to lack of epidemiological information, giving a final study total of $183(22.5 \%)$ MRSA strains considered to have caused clinical infection. The incidence of MRSA from all clinical specimens examined in the study hospitals was $0 \cdot 2 \%(183 / 107406)$. None of the subjects died in any of the MRSA cases.

By CDC criteria the 183 MRSA infections were categorized as CA-MRSA (70, 38.3\%), HA-MRSA $(45,24 \cdot 6 \%)$ and HACO-MRSA $(68,37 \cdot 2 \%)$ (Table 1). Overall, $52 \cdot 5 \%$ of all patients were female but this sex was overrepresented in HACO-MRSA infections (64.7\%) compared to CA-MRSA and HA-MRSA cases $(P=0.033)$. Two predominant age groups were evident: $0-14$ years $(38 \cdot 8 \%)$ and $20-40$ years $(39 \cdot 3 \%)$ with an older median age in HA-MRSA patients. CA-MRSA strains were most often isolated from the 0-14 years age group (52.9\%) whereas HA-MRSA strains were broadly distributed across age groups, and HACO-MRSA strains in the 20-40 years age group $(58 \cdot 8 \%)$. Significantly more elderly patients were infected with HA-MRSA strains $(P<0 \cdot 001)$.

\section{Clinical characteristics}

Table 2 shows that skin and soft tissue infection (SSTI), mastitis, trauma wounds, surgical site, and the respiratory tract were the top five sites of MRSA infections and varied in frequency according to infection category. CA-MRSA occurred most frequently in SSTI (52.9\%), while HA-MRSA infections were most associated with the respiratory tract $(22 \cdot 2 \%)$, surgical site $(20.0 \%)$ and trauma wound infections $(20 \cdot 0 \%)$. Notably, HACO-MRSA strains were most frequent in mastitis cases $(47 \cdot 1 \%)$. Most $(89 \cdot 6 \%)$ patients were treated as inpatients, primarily in general surgery $(24 \%)$, mastitis $(12 \%)$ and orthopaedic $(11 \cdot 5 \%)$ departments. However, it is noteworthy that $21.4 \%$ of outpatients were categorized as CA-MRSA infections. 
Table 1. Demographic characteristics of CA-MRSA, HA-MRSA and HACO-MRSA of case-patients

\begin{tabular}{|c|c|c|c|c|c|}
\hline Characteristics & $\begin{array}{l}\text { Total } \\
n(\%)\end{array}$ & $\begin{array}{l}\text { CA-MRSA } \\
n(\%)\end{array}$ & $\begin{array}{l}\text { HA-MRSA } \\
n(\%)\end{array}$ & $\begin{array}{l}\text { HACO-MRSA } \\
n(\%)\end{array}$ & $P$ value \\
\hline Isolates & $183(100 \cdot 0)$ & $70(38 \cdot 3)$ & $45(24 \cdot 6)$ & $68(37 \cdot 2)$ & \\
\hline Sex & & & & & $0 \cdot 033$ \\
\hline Male & $87(47 \cdot 5)$ & $40(57 \cdot 1)$ & $23(51 \cdot 1)$ & $24(35 \cdot 3)$ & \\
\hline Female & $96(52 \cdot 5)$ & $30(42 \cdot 9)$ & $22(48 \cdot 9)$ & $44(64 \cdot 7)$ & \\
\hline Median age (range) & $24(0-86)$ & $23(0-68)$ & $40(0-86)$ & $23(0-55)$ & \\
\hline $0-14$ & $71(38 \cdot 8)$ & $37(52 \cdot 9)$ & $10(22 \cdot 2)$ & $24(35 \cdot 3)$ & $0 \cdot 003$ \\
\hline $15-19$ & $5(2 \cdot 7)$ & $2(2 \cdot 9)$ & $2(4 \cdot 4)$ & $1(1 \cdot 5)$ & $0 \cdot 847$ \\
\hline $20-40$ & $72(39 \cdot 3)$ & $20(28 \cdot 6)$ & $12(26 \cdot 7)$ & $40(58 \cdot 8)$ & $<0.001$ \\
\hline $41-60$ & $23(12 \cdot 6)$ & $10(14 \cdot 3)$ & $10(22 \cdot 2)$ & $3(4 \cdot 4)$ & $0 \cdot 016$ \\
\hline$>60$ & $12(6 \cdot 6)$ & $1(1 \cdot 4)$ & $11(24 \cdot 4)$ & $0(0 \cdot 0)$ & $<0 \cdot 001$ \\
\hline
\end{tabular}

CA-MRSA, Community-associated methicillin-resistant Staphylococcus aureus; HA, hospital-associated; HACO, healthcare-associated community-onset.

Table 2. Infectious type and location of patients with CA-MRSA, HA-MRSA and HACO-MRSA

\begin{tabular}{|c|c|c|c|c|}
\hline Characteristics & $\begin{array}{l}\text { CA-MRSA } \\
n(\%)\end{array}$ & $\begin{array}{l}\text { HA-MRSA } \\
n(\%)\end{array}$ & $\begin{array}{l}\text { HACO-MRSA } \\
n(\%)\end{array}$ & $\begin{array}{l}\text { Total } \\
n(\%)\end{array}$ \\
\hline \multicolumn{5}{|l|}{ Infectious type } \\
\hline SSTI* & $37(52 \cdot 9)$ & $3(6 \cdot 7)$ & $11(16 \cdot 2)$ & $51(27 \cdot 9)$ \\
\hline Mastitis & $2(2 \cdot 9)$ & $1(2 \cdot 2)$ & $32(47 \cdot 1)$ & $35(19 \cdot 1)$ \\
\hline Trauma wound & $11(15 \cdot 7)$ & $9(20 \cdot 0)$ & $2(2 \cdot 9)$ & $22(12 \cdot 0)$ \\
\hline Surgical site & $2(2 \cdot 9)$ & $9(20 \cdot 0)$ & $8(11 \cdot 8)$ & $19(10 \cdot 4)$ \\
\hline Respiratory tract & $1(1 \cdot 4)$ & $10(22 \cdot 2)$ & $6(8 \cdot 8)$ & $17(9 \cdot 3)$ \\
\hline Blood stream & $5(7 \cdot 1)$ & $4(8 \cdot 9)$ & $4(5 \cdot 9)$ & $13(7 \cdot 1)$ \\
\hline Other & $12(17 \cdot 1)$ & $9(20 \cdot 0)$ & $5(7 \cdot 4)$ & $26(14 \cdot 2)$ \\
\hline Inpatients (department) & $55(78 \cdot 6)$ & $45(100 \cdot 0)$ & $64(94 \cdot 1)$ & $164(89 \cdot 6)$ \\
\hline General surgery & $17(24 \cdot 3)$ & $10(22 \cdot 2)$ & $17(25 \cdot 0)$ & $44(24 \cdot 0)$ \\
\hline Mastitis & $0(0 \cdot 0)$ & $2(4 \cdot 4)$ & $20(19 \cdot 4)$ & $22(12 \cdot 0)$ \\
\hline Orthopaedics & $11(15 \cdot 7)$ & $8(17 \cdot 8)$ & $2(2 \cdot 9)$ & $21(11 \cdot 5)$ \\
\hline Paediatrics & $8(11 \cdot 4)$ & $1(2 \cdot 2)$ & $5(7 \cdot 4)$ & $14(7 \cdot 7)$ \\
\hline Internal medicine & $4(5 \cdot 7)$ & $6(13 \cdot 3)$ & $4(5 \cdot 9)$ & $14(7 \cdot 7)$ \\
\hline Neonate & $0(0 \cdot 0)$ & $3(6 \cdot 7)$ & $10(14 \cdot 7)$ & $13(7 \cdot 1)$ \\
\hline Other $\dagger$ & $15(21 \cdot 4)$ & $15(33 \cdot 3)$ & $6(8 \cdot 8)$ & $36(19 \cdot 7)$ \\
\hline Outpatients & $15(21 \cdot 4)$ & $0(0 \cdot 0)$ & $4(5 \cdot 9)$ & $19(10 \cdot 4)$ \\
\hline
\end{tabular}

CA-MRSA, Community-associated methicillin-resistant Staphylococcus aureus; HA, hospital-associated; HACO, healthcare-associated community-onset.

* SSTI, Skin and soft-tissue infections (epifolliculitis, carbuncle, pustulosis, cellulitis, necrotizing lesions).

$\dagger$ MRSA patients in other departments (neurosurgery, 4.9\%; intensive care unit, 3.3\%; paediatric intensive care unit, $1 \cdot 6 \%$ ).

\section{Antimicrobial resistance}

In the three categories of infections, $>80 \%$ and $60 \%$ of strains were resistant to erythromycin and clindamycin, respectively. Compared to HA-MRSA strains, the other two categories exhibited lower resistance rates for gentamicin $(P<0 \cdot 001)$, ciprofloxacin $(P<$ $0.001)$, sulfamethoxazole/ trimethoprim $(P=0.009)$ and tetracycline $(P=0.005)$, although over $40 \%$ were resistant to tetracycline. All isolates were susceptible to vancomycin and linezolid (Supplementary Table S2).

\section{Genetic characterization}

All 183 study strains were positive for nuc and mecA genes, with $51.9 \%$ harbouring PVL genes (Table 3); the latter being more frequent in $\mathrm{CA}^{-}$and HACO-MRSA strains. One or more of 9 of the 16 
Table 3. Distribution of nuc, mec $A, P V L$ and enterotoxin genes, SCCmec and agr types in CA-MRSA, HA-MRSA and HACO-MRSA strains

\begin{tabular}{|c|c|c|c|c|c|}
\hline Characteristics & $\begin{array}{l}\text { Total } \\
n(\%)\end{array}$ & $\begin{array}{l}\text { CA-MRSA } \\
n(\%)\end{array}$ & $\begin{array}{l}\text { HA-MRSA } \\
n(\%)\end{array}$ & $\begin{array}{l}\text { HACO-MRSA } \\
n(\%)\end{array}$ & $P$ value \\
\hline$N u c$ & $183(100 \cdot 0)$ & $70(100 \cdot 0)$ & $45(100 \cdot 0)$ & $68(100 \cdot 0)$ & \\
\hline mecA & $183(100 \cdot 0)$ & $70(100 \cdot 0)$ & $45(100 \cdot 0)$ & $68(100 \cdot 0)$ & \\
\hline PVL & $95(51 \cdot 9)$ & $48(68 \cdot 6)$ & $10(22 \cdot 2)$ & $37(54 \cdot 4)$ & $<0.001$ \\
\hline Any enterotoxin gene & $160(87 \cdot 4)$ & $58(82 \cdot 9)$ & $44(97 \cdot 8)$ & $58(85 \cdot 3)$ & $0 \cdot 047$ \\
\hline sea & $11(6 \cdot 0)$ & $1(1 \cdot 4)$ & $10(22 \cdot 2)$ & $0(0 \cdot 0)$ & $<0 \cdot 001$ \\
\hline$s e b$ & $117(63 \cdot 9)$ & $43(61 \cdot 4)$ & $25(55 \cdot 6)$ & $49(72 \cdot 1)$ & $0 \cdot 168$ \\
\hline $\sec$ & $15(8 \cdot 2)$ & $8(11 \cdot 4)$ & $3(6 \cdot 7)$ & $4(5 \cdot 9)$ & $0 \cdot 485$ \\
\hline Other* & $17(9 \cdot 3)$ & $6(8 \cdot 6)$ & $6(13 \cdot 3)$ & $5(7 \cdot 4)$ & $0 \cdot 581$ \\
\hline Non-typable & $23(12 \cdot 6)$ & $12(17 \cdot 1)$ & $1(2 \cdot 2)$ & $10(14 \cdot 7)$ & $0 \cdot 047$ \\
\hline \multicolumn{6}{|l|}{ SCCmec type } \\
\hline III & $11(6 \cdot 0)$ & $0(0 \cdot 0)$ & $11(24 \cdot 4)$ & $0(0 \cdot 0)$ & $<0 \cdot 001$ \\
\hline IV & $83(45 \cdot 4)$ & $29(41 \cdot 4)$ & $20(44 \cdot 4)$ & $34(50 \cdot 0)$ & $0 \cdot 594$ \\
\hline $\mathrm{V}$ & $62(33 \cdot 9)$ & $26(37 \cdot 1)$ & $11(24 \cdot 4)$ & $25(36 \cdot 8)$ & $0 \cdot 315$ \\
\hline $\mathrm{IV} / \mathrm{V}$ & $145(79 \cdot 2)$ & $55(78 \cdot 6)$ & $31(68 \cdot 9)$ & $59(86 \cdot 8)$ & $0 \cdot 072$ \\
\hline Non-typable & $27(14 \cdot 8)$ & $15(21 \cdot 4)$ & $3(6 \cdot 7)$ & $9(13 \cdot 2)$ & $0 \cdot 086$ \\
\hline \multicolumn{6}{|l|}{$a g r$ type } \\
\hline I & $144(78 \cdot 7)$ & $54(77 \cdot 1)$ & $33(73 \cdot 3)$ & $57(83 \cdot 8)$ & $0 \cdot 393$ \\
\hline II & $10(5 \cdot 5)$ & $1(1 \cdot 4)$ & $7(15 \cdot 6)$ & $2(2 \cdot 9)$ & $0 \cdot 004$ \\
\hline III & $22(12 \cdot 0)$ & $11(15 \cdot 7)$ & $3(6 \cdot 7)$ & $8(11 \cdot 8)$ & $0 \cdot 354$ \\
\hline $\mathrm{I} / \mathrm{II}$ & $154(84 \cdot 2)$ & $55(78 \cdot 6)$ & $40(88 \cdot 9)$ & $59(86 \cdot 8)$ & $0 \cdot 261$ \\
\hline Non-typable & $7(3 \cdot 8)$ & $4(5 \cdot 7)$ & $2(4 \cdot 4)$ & $1(1 \cdot 5)$ & $0 \cdot 438$ \\
\hline
\end{tabular}

CA-MRSA, Community-associated methicillin-resistant Staphylococcus aureus; HA, hospital-associated; HACO, healthcare-associated community-onset.

* Other enterotoxin genes $(<3 \%)$.

enterotoxin genes tested were detected in $87.4 \%$ of the strains (Table 3) with seb (63.9\%) being the most dominant in each strain category.

The majority $(82 \cdot 5 \%)$ of 183 strains could be allocated to a SCCmec type with types IV (45.4\%) and $\mathrm{V}(33.9 \%)$ being the most prevalent. No difference in SCCmec IV/V presence was evident between the three categories $(P=0 \cdot 07)$ (Table 3$)$. By MLST, 31 STs were identified of which nine were novel to this study. The STs fell into 14 CCs with one singleton (Supplementary Table S3). CC59 (60.7\%), predominated in each category followed by $\mathrm{CC} 1(7 \cdot 7 \%)$ and CC239 (6.6\%). Forty-four different spa types were found and four were novel; type 4437 accounted for $51.9 \%$ of all study strains and far exceeded the frequency of the second most common type, t114 $(4 \cdot 9 \%)$. Likewise, $a g r$ type I (78.7\%) predominated in all strains within each group; agr type IV was not detected, and $3 \cdot 8 \%$ were non-typable (Table 3 ).

Table 4 shows $36 \cdot 1 \%$ of all strains in six clonal groups according to the USA clonal groupings. USA1000 was represented by $38(20 \cdot 8 \%)$ strains followed by USA400 (8.7\%) with no significant difference between the categories of infections, but 117 of the total strains were characterized by PFGE profiles distinct from the USA groups.

The predominant clone in CA-, HA- and HACOMRSA was defined as CC59-t437-SCCmec IV/Vagr I with a distribution of $51 \cdot 4 \%$ (36/70), $28 \cdot 9 \%$ $(13 / 45)$ and $52 \cdot 9 \%$ (36/68), respectively. The specific subclone USA1000 within this genetic lineage was identified in $12.9 \%$ of CA-, $20 \%$ of HA- and $10.3 \%$ of HACO-MRSA strains (Table 5).

\section{DISCUSSION}

We performed epidemiological and molecular characterization of 183 clinical MRSA isolates from patients attending nine sentinel hospitals in Shenzhen in 2014 who were categorized as having CA-, HA-, or HACO-MRSA. The top three clones identified were CC59-t437-SCCmec IV/V-agr I (46.4\%), which was dominant across each infection category, CC239t030/t037-SCCmec III-agr I (4.4\%), and CC1-t114SCCmec IV-agr III (3.8\%). Older patients were more likely to harbour HA-MRSA strains and the major 


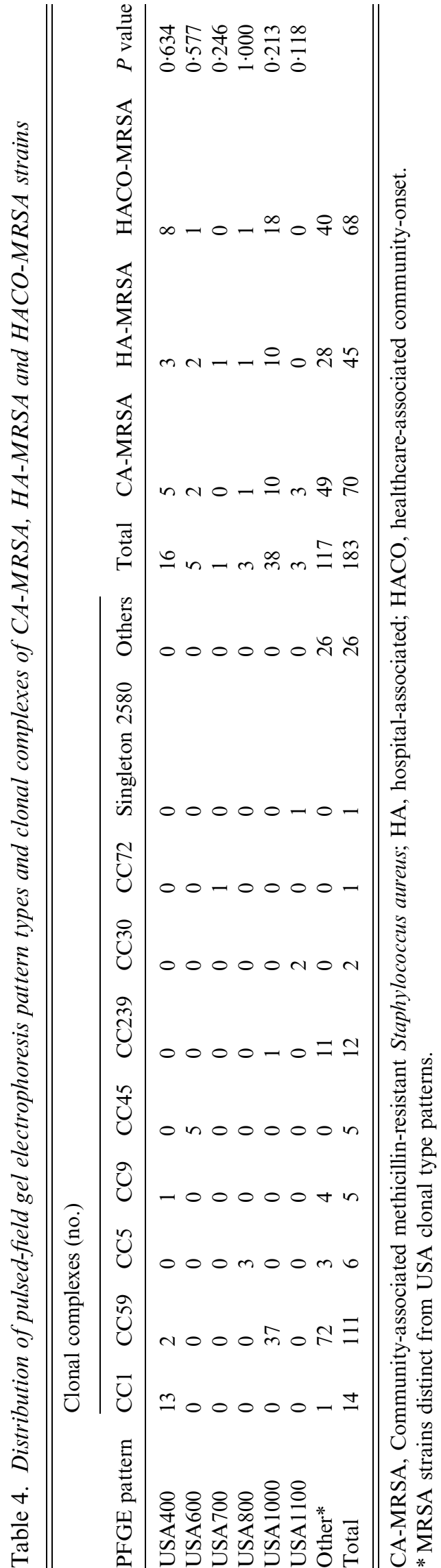

infection sites varied with the category of infection. Overall, our findings suggest that the MRSA strains of different origins shared similar major molecular features but differed by demographic and clinical characteristics of the patient cohorts.

MRSA strains from the three patient groups exhibited high rates of resistance to erythromycin, clindamycin and tetracycline, and HA-MRSA isolates in particular were often also resistant to gentamicin and ciprofloxacin. The antimicrobial resistance profile of the primary clone CC59-t437-SCCmec IV/ $\mathrm{V}-a g r$ I strains was consistent with that previously reported for CC59 strains in another study in mainland China [19]. Knowledge of drug resistance rates for MRSA strains is essential for targeting of effective antimicrobial drug therapy and can help to reduce the likelihood of the emergence of other resistant pathogens.

In China, CC59-t437-SCCmec IVa was the most predominant CA-MRSA clone in neonates [20], while CC239-t030/t037-SCCmec III was most frequently recovered from hospital patients during the past year [21]. An earlier study of MRSA strains from nine teaching hospitals in six cities between 2009 and 2012 in Guangzhou, Guangdong Province, China, showed that CC239-t030/t037-SCCmec III was the most predominant MRSA clone [22], but epidemiological classification of patient category was not conducted. Another investigation undertaken 5 years previously over 6 months also identified the latter as the dominant clone in Guangzhou as an HA-MRSA lineage but also reported the isolation of clone CC59-t437-SCCmec IV which is classified as a CAMRSA lineage [23]. Moreover, a recent comprehensive review of MRSA data from Asian countries demonstrated that in Hong Kong and Taiwan, the major clonal lineage of CA-MRSA strains was ST59-SCCmec IV/V, and the major clone of HAMRSA strains was ST239-SCCmec III [24]. In contrast with earlier studies in Hong Kong, Taiwan, and mainland China, the CC59-t437-SCCmec IV/V clone appears to have replaced CC239-t030/t037SCCmec III to become the major HA-MRSA clone in our study. This indicates that CA-MRSA strain lineages may be replacing traditional HA-MRSA strains, as illustrated here by the possible spread of the CC59-t437-SCCmecIV/V clone from the community into hospitals in Shenzhen. A possible similar migration of MRSA strains from the community into hospitals was reported for clone ST30-SCCmec IV-t019 which was the initially prevalent clone of 
Table 5. Distribution of CC59-t437-SCCmec IVIV-agr I in CA-MRSA, HA-MRSA and HACO-MRSA strains

\begin{tabular}{|c|c|c|c|c|c|c|}
\hline Category & $\mathrm{CC}(n)$ & $\mathrm{ST}(n)$ & spa $(n)$ & SCCmec type (n) & $a g r$ type $(n)$ & PFGE pattern $(n)$ \\
\hline \multirow[t]{6}{*}{ CA-MRSA } & $59(36)$ & $59(9)$ & t437 (9) & IV (8), V (1) & I (9) & USA1000 (9) \\
\hline & & 59 (13) & t437 (13) & IV (6), V (7) & I (13) & Other (13)* \\
\hline & & $338(11)$ & t437 (11) & V (11) & I (11) & Other (11) \\
\hline & & $2961(1)$ & t437 (1) & V (1) & I (1) & Other (1) \\
\hline & & $2962(1)$ & t437 (1) & $\mathrm{V}(1)$ & I (1) & Other (1) \\
\hline & & 3189 (1) & t437 (1) & IV (1) & I (1) & Other (1) \\
\hline \multirow[t]{4}{*}{ HA-MRSA } & $59(13)$ & 59 (7), 3031 (1) & t437 (8) & IV (8) & I (8) & USA1000 (8) \\
\hline & & $338(1)$ & t437 (1) & $\mathrm{V}(1)$ & I (1) & USA1000 (1) \\
\hline & & 59 (1), 338 (2) & t437 (3) & V (3) & I (3) & Other (3) \\
\hline & & $59(1)$ & t437 (1) & IV (1) & I (1) & Other (1) \\
\hline \multirow[t]{5}{*}{ HACO-MRSA } & $59(36)$ & $59(6)$ & t437 (6) & IV (4), V (2) & I (6) & USA1000 (6) \\
\hline & & $3191(1)$ & t437 (1) & IV (1) & I (1) & USA1000 (1) \\
\hline & & $59(17)$ & t437 (17) & IV (9), V (8) & I (17) & Other (17) \\
\hline & & $338(11)$ & t437 (11) & V (11) & I (11) & Other (11) \\
\hline & & $2982(1)$ & $\mathrm{t} 437(1)$ & IV (1) & I (1) & Other (1) \\
\hline
\end{tabular}

CC, Clonal complex; ST, sequence type; CA-MRSA, community-associated methicillin-resistant Staphylococcus aureus; HA, hospital-associated; HACO, healthcare-associated community-onset.

* MRSA strains distinct from USA clonal type patterns.

CA-MRSA but was also found in HA-MRSA strains in the Philippines [25]. Such community-hospital transmission may have occurred through healthcare workers and possibly the environment of patients discharged from hospitals. This underlines the need for hospitals to implement routine screening of patients for MRSA colonization on admission to hospital, and undertake active surveillance for MRSA carriage in 'patient-facing' staff.

The globally predominant CA-MRSA clones are ST1-IV (WA-1, USA400), ST5-IV (Argentina), ST8IV (USA300), ST30-IV (South West Pacific clone), ST59-V (Taiwan clone), ST72-IV (Korea clone) and ST80-IV (European clone) [26]. However, in our study, the lineages that were previously most commonly associated with CA-MRSA were found in each of the three categories. This reinforces our view that transmission events from community to hospital patients are important factors that can affect the composition of each category, which in turn may contribute to evolutionary changes in the molecular characteristics of dominant strains. Such a scenario occurred with SCCmec type III which was by far the most prevalent mec element $(87 \cdot 3 \%)$ in HA-MRSA strains isolated in the Chinese mainland between 2001 and 2010 [27], but our findings suggest that it is being replaced by traditional CA-MRSA SCCmec types IV/V. Such a change may contribute to increased fitness of strains in hospital environments.
PFGE typing showed that approximately one-fifth of MRSA study strains were USA1000, which was one of the major CA-MRSA clones circulating in Taiwan [28], while the second predominant type, USA400, is a traditional CA-MRSA clone [29]. This finding underlines their prevalence in communities and hospitals. It has been well documented that $\mathrm{CC} 1$ isolates correspond to USA400 and CC59 to USA1000 [30], but in our study USA400 was associated not only with $\mathrm{CC} 1(n=13)$, but also CC59 $(n=2)$ and CC9 $(n=1)$, and USA1000 with CC59 $(n=37)$ and CC239 $(n=1)$. This further supports the view that the traditional relationship between CCs and PFGE clonal groups is undergoing gradual change. Additionally, across all infection categories, $63.9 \%$ of MRSA strains did not belong to USA100800,1000 and 1100 , which provides a diverse background to give a context for the proliferation of the predominant clone, CC59-t437-SCCmec IV/V-agr, I across Shenzhen hospitals.

Others have reported that intensive-care units (ICUs) are high-risk areas with a high level of endemic MRSA [31-33]. However, in our study, MRSA infections were commonly identified in departments of general surgery, mastitis and orthopaedics, and $<5 \%$ of cases occurred in the ICU setting, suggesting that we may have a different clinical distribution of cases compared to some countries, and indicates a need to focus more on departments other than ICUs. 
An apparently unique observation of this study was that HACO-MRSA strains were most commonly isolated from patients with mastitis. Of 20 such strains, 13 were isolated from pus samples from nursing mothers in a specialist maternity and child healthcare hospital. Nine of these strains were of CC59-t437SCCmec IV/V-agr I but PFGE typing showed no relationship between these or others. The telephone survey confirmed that these mothers took at least 2-3 months to recover from the infection and this seriously affected breastfeeding. Such cases therefore have a high risk of transmitting infections to their babies, and it would seem useful to implement screening of oral MRSA colonization in nursing mothers' babies as an infection control measure in the future. Additionally, two HA-MRSA strains originated from mastitis cases who were not nursing mothers but molecular typing showed that the strains were CC59-t437-SCCmec V-agr I and CC59-t14776SCCmec IV-agr I, which were similar to the strains isolated from the nursing mothers memtioned above.

Our study is subject to some limitations. First, there are more than 90 hospitals in Shenzhen; we only included nine sentinel hospitals which may not be fully representative of all MRSA patients in Shenzhen hospitals. Second, it is possible that not all doctors used the same criteria when requesting bacterial cultures, so some cases could have been missed. Third, although a total of 812 MRSA strains were obtained, only 183 strains were analysed due to exclusion of colonizing strains and specimens with incomplete information, which could also have biased the results.

In conclusion, in our sentinel hospital population, CA-, HA- and HACO-MRSA infections were caused predominantly by a common clone which exhibits combined characteristics of both CA-MRSA (CC59t437-SCCmec IV/V) and HA-MRSA (agr I) lineages, but patients differed in demographic and clinical characteristics. This highlights the possible transmission of MRSA between community and hospitals. For MRSA prevention and control, hospitals should enhance surveillance in high-risk patients and healthcare personnel in clinical departments in addition to ICUs. Considering the high incidence of HACO-MRSA infections observed in nursing mothers, hospitals should implement oral MRSA screening of babies to stem mother-to-child transmission. Finally, continuous surveillance of the epidemiology and documentation of the changing aetiology of MRSA strains is necessary for the effective treatment of cases and control of transmission events.

\section{SUPPLEMENTARY MATERIAL}

For supplementary material accompanying this paper visit http://dx.doi.org/10.1017/S0950268816001552.

\section{ACKNOWLEDGEMENTS}

We thank the personnel from sentinel hospitals for their enthusiastic participation and contribution to our work. We thank Dr Linda K. McDougal from US CDC for providing PFGE protocol and BDL files of USA100-800, 1000 and 1100. We thank Dr Shanyou $\mathrm{Yu}$ from the First Affiliated Hospital of Wenzhou Medical University for providing standard SCCmec I-V strains. Dr Jeanette Rainey and Jill Shull are acknowledged for revising the manuscript.

This study was supported by Cooperative Agreement no. 5U2GGH000018 from the US Centers for Disease Control and Prevention, Technology Major Projects Foundation in China (no. 2012ZX10004215-003-005) and the Shenzhen Municipal Science and Technology Programme (CXZZ20140411105636301).

The findings and conclusions in this report are those of the authors and do not necessarily represent the official position of the US CDC.

\section{DECLARATION OF INTEREST}

None.

\section{REFERENCES}

1. Stryjewski ME, Corey GR. Methicillin-resistant Staphylococcus aureus: an evolving pathogen. Clinical Infectious Diseases 2014; 58 (Suppl. 1): S10-19.

2. Thompson RL, Cabezudo I, Wenzel RP. Epidemiology of nosocomial infections caused by methicillin-resistant Staphylococcus aureus. Annals of Internal Medicine 1982; 97: 309-317.

3. Chambers HF. The changing epidemiology of Staphylococcus aureus? Emerging Infectious Diseases 2001; 7: 178-182.

4. Moran GJ, et al. Methicillin-resistant S. aureus infections among patients in the emergency department. New England Journal of Medicine 2006; 355: 666-674.

5. Klevens RM, et al. Invasive methicillin-resistant Staphylococcus aureus infections in the United States. Journal of the American Medical Association 2007; 298: 1763-1771.

6. Naimi TS, et al. Comparison of community- and health care-associated methicillin-resistant Staphylococcus aureus infection. Journal of the American Medical Association 2003; 290: 2976-2984.

7. Benoit SR, et al. Community strains of methicillinresistant Staphylococcus aureus as potential cause of 
healthcare-associated infections, Uruguay, 2002-2004. Emerging infectious diseases 2008; 14: 1216-1223.

8. Lee SM, et al. Fitness cost of staphylococcal cassette chromosome mec in methicillin-resistant Staphylococcus aureus by way of continuous culture. Antimicrobial Agents and Chemotherapy 2007; 51: 1497-1499.

9. Chua K, et al. Antimicrobial resistance: not communityassociated methicillin-resistant Staphylococcus aureus (CA-MRSA)! A clinician's guide to community MRSA - its evolving antimicrobial resistance and implications for therapy. Clinical Infectious Diseases 2011; 52: $99-114$.

10. Otter JA, French GL. Community-associated meticillinresistant Staphylococcus aureus: the case for a genotypic definition. Journal of Hospital Infection 2012; 81: 143-148.

11. Clinical and Laboratory Standards Institute. Performance standards for antimicrobial susceptibility testing; twenty-third informational supplement, M100-S23. Clinical and Laboratory Standards Institute, Wayne, PA 2013.

12. McDonald RR, et al. Development of a triplex real-time PCR assay for detection of Panton-Valentine leukocidin toxin genes in clinical isolates of methicillin-resistant Staphylococcus aureus. Journal of Clinical Microbiology 2005; 43: 6147-6149.

13. Shi XL, et al. Application of real time fluorescent PCR technique in typing and screening of staphylococcal enterotoxin. Chinese Journal of Health Laboratory Technology 2011; 21: 1715-1717.

14. Chen L, et al. Multiplex real-time PCR for rapid staphylococcal cassette chromosome mec typing. Journal of Clinical Microbiology 2009; 47: 3692-3706.

15. Enright MC, et al. Multilocus sequence typing for characterization of methicillin-resistant and methicillinsusceptible clones of Staphylococcus aureus. Journal of Clinical Microbiology 2000; 38: 1008-1015.

16. Strommenger B, et al. spa Typing of Staphylococcus aureus as a frontline tool in epidemiological typing. Journal of Clinical Microbiology 2008; 46: 574-581.

17. Peacock SJ, et al. Virulent combinations of adhesin and toxin genes in natural populations of Staphylococcus aureus. Infection and Immunity 2002; 70: 4987-4996.

18. McDougal LK, et al. Pulsed-field gel electrophoresis typing of oxacillin-resistant Staphylococcus aureus isolates from the United States: establishing a national database. Journal of Clinical Microbiology 2003; 41: 5113-5120.

19. Li J, et al. Molecular and clinical characteristics of clonal complex 59 methicillin-resistant Staphylococcus aureus infections in Mainland China. PLOS ONE 2013; 8: e70602.

20. Li S, et al. Clinical and molecular characteristics of community-acquired methicillin-resistant Staphylococcus aureus infections in Chinese neonates. Acta Pathologica Microbiologica et Immunologica Scandinavica 2015; 123: 28-36.
21. Wu D, et al. Epidemiology and molecular characteristics of community-associated methicillin-resistant and methicillin-susceptible Staphylococcus aureus from skin/soft tissue infections in a children's hospital in Beijing, China. Diagnostic Microbiology and Infectious Disease 2010; 67: 1-8.

22. Cheng H, et al. Molecular and phenotypic evidence for the spread of three major methicillin-resistant Staphylococcus aureus clones associated with two characteristic antimicrobial resistance profiles in China. Journal of Antimicrobial Chemotherapy 2013; 68: 2453-2457.

23. Cheng $\mathbf{H}$, et al. Molecular typing and antimicrobial susceptibility of methicillin-resistant Staphylococcus aureus isolated from Guangzhou, China. Journal of Third Military Medical University 2013; 35: 696-701.

24. Chen CJ, Huang YC. New epidemiology of Staphylococcus aureus infection in Asia. Clinical Microbiology and Infection 2014; 20: 605-623.

25. Song JH, et al. Spread of methicillin-resistant Staphylococcus aureus between the community and the hospitals in Asian countries: an ANSORP study. Journal of Antimicrobial Chemotherapy 2011; 66: 1061-1069.

26. Mediavilla JR, et al. Global epidemiology of community-associated methicillin resistant Staphylococcus aureus (CA-MRSA). Current Opinion in Microbiology 2012; 15: 588-595.

27. Chao G, et al. Phenotypic and genotypic characterization of methicillin-resistant Staphylococcus aureus (MRSA) and methicillin-susceptible Staphylococcus aureus (MSSA) from different sources in China. Foodborne Pathogens and Disease 2013; 10: 214-221.

28. Boyle-Vavra S, et al. Successful multiresistant community-associated methicillin-resistant Staphylococcus aureus lineage from Taipei, Taiwan, that carries either the novel Staphylococcal chromosome cassette mec (SCCmec) type VT or SCCmec type IV. Journal of Clinical Microbiology 2005; 43: 4719-4730.

29. Pardos de la Gandara M, et al. Molecular types of MRSA and MSSA strains causing skin and soft tissue infections and nasal colonization identified in community health centers in New York City. Journal of Clinical Microbiology 2015; 53: 2648-2658.

30. Yeung M, et al. Identification of major clonal complexes and toxin producing strains among Staphylococcus aureus associated with atopic dermatitis. Microbes and Infection 2011; 13: 189-197.

31. Girou E, et al. Selective screening of carriers for control of methicillin-resistant Staphylococcus aureus (MRSA) in high-risk hospital areas with a high level of endemic MRSA. Clinical Infectious Diseases 1998; 27: 543-550.

32. Lucet JC, et al. Prevalence and risk factors for carriage of methicillin-resistant Staphylococcus aureus at admission to the intensive care unit: results of a multicenter study. Archives of Internal Medicine 2003; 163: 181-188.

33. Thompson DS. Methicillin-resistant Staphylococcus aureus in a general intensive care unit. Journal of the Royal Society of Medicine 2004; 97: 521-526. 